\title{
Optimizing the management of intermediate-stage hepatocellular carcinoma: Current trends and prospects
}

\author{
Takuji Torimura and Hideki Iwamoto \\ Division of gastroenterology, Department of Medicine, Kurume University School of Medicine, Research Center for Innovative Cancer \\ Therapy Kurume University, Kurume, Japan
}

Hepatocellular carcinoma (HCC) is usually accompanied by chronic liver damage, which sometimes influences the selection of HCC treatment. The Barcelona Clinic Liver Cancer (BCLC) staging system, which was first introduced in 1999, is the most commonly used worldwide. Although the intermediate-stage (BCLC stage B) includes the largest number and heterogeneous HCC patients, the recommended treatment option is transarterial chemoembolization (TACE) only. However, recent progress in radical treatments such as hepatic resection, liver transplantation, radiation therapy, and percutaneous therapy has made it possible to treat selected patients with BCLC stage B HCC. Radical treatments are expected to prolong survival time. To-date, TACE has also progressed. In addition to conventional TACE, balloon-occluded TACE and drug-eluting beads TACE are available. These new modalities of TACE will improve therapeutic efficacy and reduce adverse events. One of the most serious concerns of TACE is that repeated TACE reduces the treatment effect and induces liver function impairment. The decision on when TACE should be interrupted is complex. Many molecular targeted agents are now available, and immune checkpoint inhibitors will soon be available for HCC patients with ChildPugh class A worldwide. Under these circumstances, in patients with TACE unsuitability, switching to molecular targeted agents before deterioration of liver function might improve the prognosis compared to repeated TACE. We should pay attention to stop TACE in TACE-unsuitable HCC patients as it can induce the deterioration of liver function. (Clin Mol Hepatol 2021;27:236-245)

Keywords: Carcinoma, Hepatocellular; BCLC; Chemoembolization, Therapeutic; Molecular targeted therapy; Immune checkpoint inhibitors

\begin{abstract}
Abbreviations:
ALBI, albumin-bilirubin; B-TACE, balloon-occluded transarterial

chemoembolization; BCLC, Barcelona Clinic Liver Cancer; CR, complete response; CTACE, conventional transarterial chemoembolization; DCs, dendritic cells; DEB-TACE, drug-eluting beads transarterial chemoembolization; ECOG, Eastern Cooperative Oncology Group; FDA, Food and Drug Administration; HCC, hepatocellular carcinoma; ICIs, immune checkpoint inhibitors; MRECIST, modified response evaluation criteria in solid tumors; MTAs, molecular targeted agents; OS, overall survival; PD-1, programmed cell death protein-1; PD-L1, programmed death-ligand-1; PDGFR, platelet-derived growth factor receptor; PS, performance status; RCT, randomized controlled trial; RFA, radiofrequency ablation; SBRT, stereotactic body radiotherapy; TACE, transarterial chemoembolization; TARE, transarterial radioembolization; VEGF, vascular endothelial growth factor
\end{abstract}

\section{Corresponding author : Takuji Torimura}

Division of gastroenterology, Department of Medicine, Kurume University School of Medicine, Research Center for Innovative Cancer Therapy Kurume University, 67 Asahi-machi, Kurume city 830-0011, Japan Tel: +81-942-31-7561, Fax: +81-942-34-2623

E-mail: tori@med.kurume-u.ac.jp

https://orcid.org/0000-0002-4863-4278 


\section{INTRODUCTION}

Liver cancer is the third leading cause of cancer-related death and ranks the sixth most common neoplasm, with 841,080 diagnosed and 781,631 deaths globally in 2018 ${ }^{1,2}$ These numbers are gradually increasing. Hepatocellular carcinoma (HCC) accounts for the majority of primary liver cancers. The majority of HCC develops in Asian countries. ${ }^{3}$ Therefore, HCC is a significant health threat in Asian countries. HCC has two unique characteristics. One is that it usually develops from the chronically damaged liver. The other is that HCC repeatedly shows a multi-centric recurrence after curative treatment. Recently, several radical and non-radical treatments have been developed for HCC. Prognostic assessment and treatment allocation are crucial steps in the management of patients with HCC. Since most patients with HCC are associated with chronic liver diseases, a staging system with the information of tumor burden and liver-function reserve has been proposed.
The Barcelona Clinic Liver Cancer (BCLC) staging system has been extensively validated and is the most commonly used. The $B C L C$ stage $B$ is quite broad and includes a heterogeneous patient population. The recommended treatment is transarterial chemoembolization (TACE) only. There is growing evidence that more aggressive radical treatments such as hepatic resection and radiofrequency ablation (RFA) are feasible for selected HCC patients with BCLC stage B. ${ }^{4}$ Recently, molecular targeted agents (MTAs) have become available for BCLC stage B and C HCC patients with Child-Pugh class $A^{5}{ }^{5}$ In addition, atezolizumab (anti-programmed death-ligand-1 [PD-L1] antibody) plus bevacizumab (anti-vascular endothelial growth factor [VEGF] antibody) has proven to be the superior to sorafenib in phase 3 randomized controlled trial (RCT). ${ }^{6}$ This combination treatment is available for BCLC stage $B$ and $C$ HCC patients. As these new systemic therapies are restricted to HCC patients with Child-Pugh class A, another treatment will be required for $B C L C$ stage $B$ HCC patients with Child-Pugh class $B$.

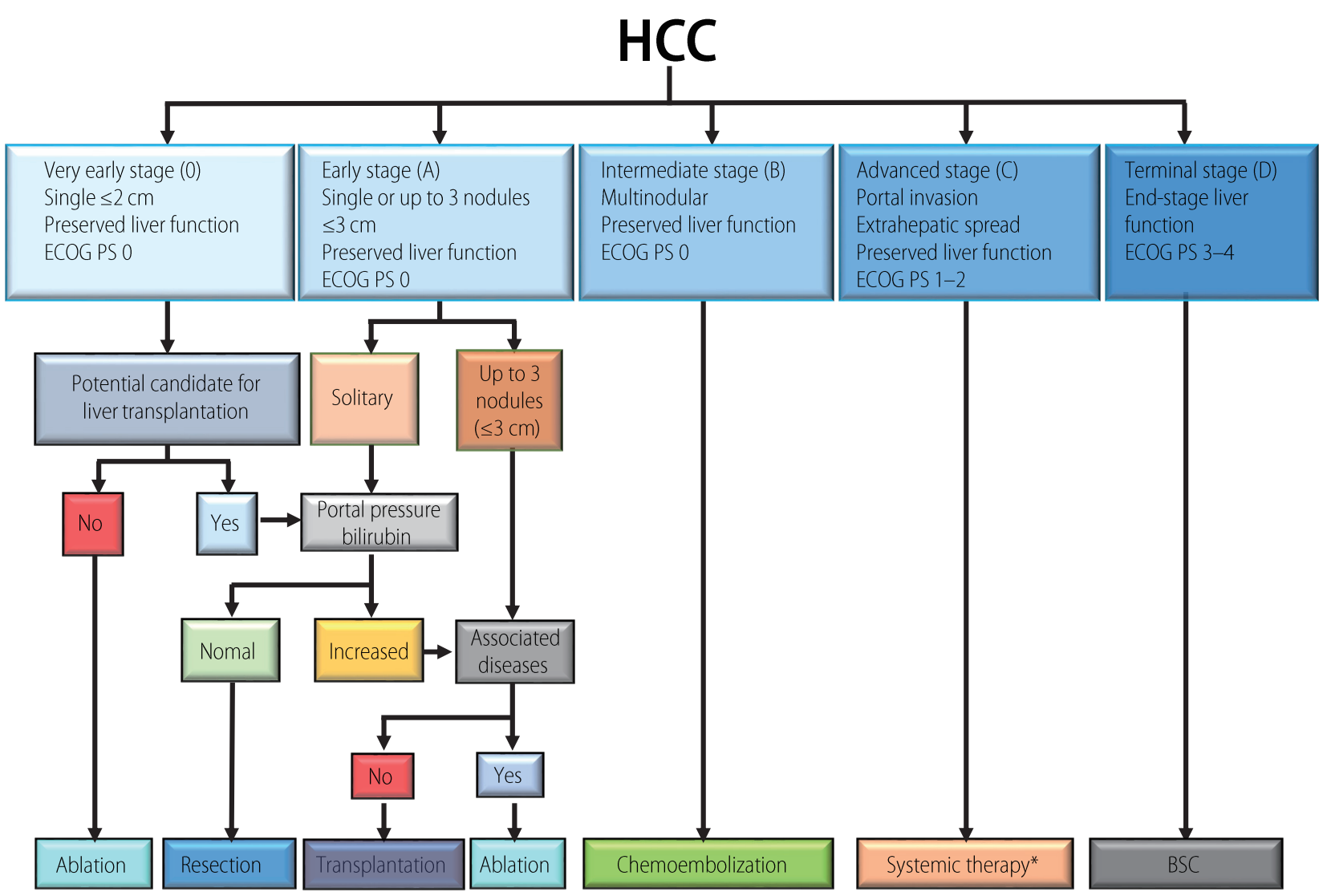

Figure 1. Barcelona Clinic Liver Cancer (BCLC) staging and treatment strategy. Modified from Forner et al. ${ }^{8}$ with permission from Elsevier. HCC, hepatocellular carcinoma; ECOG, Eastern Cooperative Oncology Group; PS, performance status; BSC, best supportive care. *Currently, sorafenib followed by regorafenib has been shown to be effective. Lenvatinib has been shown to be non-inferior to sorafenib, but no second-line option after Lenvatinib has been explored. 
There seems to be an increasing unmet need between guidelinerecommended therapy and recent evidence-based treatment in BCLC stage B HCC. In this review, we will raise the problems at the moment and discuss to fill the gap between guideline-recommended therapy and recent evidence-based treatment in BCLC stage B HCC.

\section{BRIEF REVIEW OF BCLC STAGING SYSTEM}

In 1999, the BCLC staging system was first introduced. ${ }^{7}$ The latest $B C L C$ staging system consists of five stages $(0$, very earlystage; A, early-stage; B, intermediate-stage; C, advanced-stage; $D$, terminal-stage) (Fig. 1). Among five stages, BCLC stage 0 (single nodule $<2 \mathrm{~cm}$ in diameter, Child-Pugh class A, Eastern Cooperative Oncology Group [ECOG] performance status [PS] 0) or A ( $\leq 3$ nodules $<3 \mathrm{~cm}$ in diameter, Child-Pugh class A or B, PS 0) HCC is a candidate for curative treatment (i.e., hepatic resection, RFA, transplantation). In these stages, the estimated survival time is 5 years or more. BCLC stage B (nodules out of Milan criteria without vascular invasion or extrahepatic metastasis, Child-Pugh class A or B, PS 0 ) is a candidate for TACE. The estimated survival time is more than 2.5 years. BCLC stage $C$ comprises advanced HCC with vascular invasion, extrahepatic metastasis, and mild cancer-related symptoms or both (ECOG PS 1-2). Today, these patients are usually treated with MTAs. The estimated survival time is more than 1 year. HCC patients with BCLC stage D have a poor liver function (Child-Pugh class $C$ ) and severe cancer-related symptoms (ECOG PS 3-4). The estimated survival time is only 3 months. ${ }^{8}$

\section{SUBCLASSIFICATION OF BCLC STAGE B}

The Bolondi criteria subdivided BCLC stage B based on liver function, tumor burden and ECOG PS. ${ }^{9}$ Subsequent studies have proposed various novel subclassification systems. In some of them, radical treatments were recommended as treatment options for patients with $\mathrm{BCLC}$ stage $\mathrm{B} \mathrm{HCC}{ }^{10}$ In a retrospective analysis of 80 patients with BCLC stage B HCC, Ciria et al. ${ }^{11}$ revealed that the 5 -year survival rate was higher in stage $B 1$ than in stages B2 and B3-4. They proposed the selection of hepatic resection for stage B1. As mentioned, few high-level studies are evaluating the therapeutic efficacy of percutaneous treatment or radiation therapy in patients with BCLC stage B HCC. In addition, as radical treatments sometimes require highly complex technical procedures, the selection criteria of each radical treatment for BCLC stage B HCC will be institution-dependent. However, expanding the indications for radical treatments and adding radical treatments to the first option will improve the survival benefit of patients with BCLC stage B HCC. It would be necessary to standardize the allocation of patients with BCLC stage B HCC for each radical treatment.

\section{POSSIBLE CURATIVE INTERVENTIONS FOR BCLC STAGE B HCC}

\section{Hepatic resection}

The optimal candidate for hepatic resection is HCC patients with Child-Pugh class A, without clinical signs of portal hypertension and limited tumor burden. ${ }^{9}$ In RCT comparing hepatic resection and TACE for HCC beyond Milan criteria, Yin et al. ${ }^{4}$ reported that the 1-, 2-, and 3-year overall survival rate were $76.1 \%$, $63.5 \%$, and $51.5 \%$, respectively, for hepatic resection group compared with $51.8 \%, 34.8 \%$, and $18.1 \%$, respectively, for TACE group $(P<0.001)$. In addition, a meta-analysis utilizing one RCT and high-quality nonrandomized studies revealed that a significant survival benefit was shown for hepatic resection (hazard ratio, 0.53 ; 1-year survival rate for hepatic resection vs. TACE: $84 \%$ vs. $68 \%$; 5 -year survival rate for hepatic resection vs. TACE: $45 \%$ vs. $23 \%$; respectively), suggesting that hepatic resection should be considered as a therapeutic option tailored to a carefully selected group of BCLC stage B HCC patients with well-preserved liver function. ${ }^{12}$

\section{Liver transplantation}

The Milan criteria (single tumor $\leq 5 \mathrm{~cm}$ or 3 nodules $\leq 3 \mathrm{~cm}$ ) are the most common criteria worldwide. Five-year survival rate and 5 -year recurrence rates within the Milan criteria were $71.3 \%$ and $12.3 \%$, respectively. ${ }^{13}$ The advancement of surgical techniques made it possible for liver transplantation beyond the Milan criteria. Five-year survival rates within the Asan criteria (nodule $\leq 5$ $\mathrm{cm}$, number of nodules $\leq 6$ ), University of California San Francisco criteria (single nodule $\leq 6.5 \mathrm{~cm}$, or 3 nodules $\leq 4.5 \mathrm{~cm}$ with total tumor diameter $\leq 8 \mathrm{~cm}$ ), up-to-7 criteria (the sum of maximum tumor diameter and number $<7$ ) were $70.9 \%,{ }^{13,14} 80.9 \%,{ }^{15}$ and $71.2 \%,{ }^{13}$ respectively. These findings imply that liver transplanta- 
Takuji Torimura, et al. Current treatment strategies for BCLC stage B HCC

tion is a treatment option for selected patients with $B C L C$ stage $B$ HCC. However, the available donor organ shortage is still a critical problem worldwide.

\section{Percutaneous treatment}

A retrospective study with $254 \mathrm{BCLC}$ stage B HCC patients showed that RFA demonstrated a survival benefit at 1-year compared with locoregional treatment (mostly TACE). ${ }^{16}$ In addition, a retrospective multi-center study showed the superiority of prognosis in curative treatment, including liver transplantation, hepatic resection, RFA, and percutaneous ethanol injection therapy after the adjustment for all of the confounding factors. ${ }^{17}$ However, these were all retrospective studies. There is no RCT or metaanalysis study comparing the therapeutic efficacy of percutaneous treatment and that of TACE. Therefore, it is questionable to conclude the therapeutic benefit of percutaneous treatment in selected HCC patients with BCLC stage $B$.

\section{Radiation therapy}

Stereotactic body radiotherapy (SBRT) has emerged as a good treatment option for $\mathrm{HCC}^{18}$ Comparative studies were conducted to compare the therapeutic efficacy of SBRT and RFA. ${ }^{19,20}$ However, the results were controversial. In a systematic review evaluating the therapeutic effect of SBRT for HCC less than $5 \mathrm{~cm}$ in median diameter, SBRT showed high local control and good overall survival which is compatible with RFA and hepatic resection. ${ }^{18}$ In addition, SBRT was associated with low levels of early and late toxicities. Kim et al. ${ }^{21}$ compared the therapeutic effect of SBRT and RFA with a propensity score matching technique and concluded that SBRT could be an alternative treatment to RFA, especially for larger HCCs $(>3 \mathrm{~cm})$ in a subphrenic location. These findings imply that SBRT could be a radical treatment option for selected BCLC stage B HCC patients. However, to confirm the benefits of SBRT and provide evidence for its use as a treatment option for selected BCLC stage B HCC patients, high-level RCTs are indispensable.

Transarterial radioembolization (TARE) is a catheter-based intervention where radioactive beads loaded with a beta-emitter Yttrium-90 are injected into the artery that supplies the HCC. ${ }^{22}$ The overall survival (OS) is comparable between TACE and TARE. ${ }^{23}$ Recently, two phase 3 trials in Europe (SARAH) ${ }^{24}$ and in Asia-Pacific lesion (SIRveNIB) ${ }^{25}$ showed TARE to be associated with higher response rate, longer time to progression, and lower adverse events. However, TARE failed to improve OS compared with sorafenib in BCLC stage B and C. At the European Society for Medical Oncology Asia meeting in 2018, TARE was recommended as an alternative treatment for TACE as first-line treatment for HCC patients and as a treatment for TACE-failed HCC patients with $\mathrm{BCLC}$ stage $\mathrm{B}^{26}$

\section{RECENT PROGRESS OF TACE AND SYSTEMIC THERAPY FOR BCLC STAGE B HCC}

\section{Conventional TACE (CTACE)}

The TACE technique was first developed and reported by Yamada et al. ${ }^{27}$ in 1978. In 2002, two RCTs demonstrated the survival benefit of TACE. ${ }^{28,29}$ In addition, a meta-analysis showed an improved 2-year survival rate in HCC patients treated with TACE compared with conservative managements. ${ }^{30}$ These high-grade evidence reports support the recognition of TACE as a standard treatment for patients with BCLC stage B HCC. The current median survival exceeds $30-40$ months. ${ }^{31}$ Although TACE is the only recommended standard treatment for $\mathrm{BCLC}$ stage $\mathrm{B} \mathrm{HCC}$, the applicability of TACE in BCLC stage B is $50 \%$. $^{32}$ The good candidates for TACE are asymptomatic limited multifocal or solitary HCCs that are not indications of radical treatments with well-preserved liver function (Child-Pugh score <8). ${ }^{28,33}$ Absolute and relative contraindications are as follows: large $\mathrm{HCC} \geq 10 \mathrm{~cm}$ in diameter, impairment of liver function, vascular invasion, or extrahepatic spread. ${ }^{34}$ There is no properly evaluated comparative study of survival between on-demand TACE, that is, in cases of incomplete response to the previous TACE or appearance of new lesions and TACE with regular intervals. However, on-demand TACE has become the standard treatment, because excessive TACE induces complications and liver function impairment. ${ }^{34}$

Modified response evaluation criteria in solid tumors (mRECIST), which recognizes TACE induced tumor necrosis, as indicated by the absence of a contrast agent within the tumor, demonstrated a significant association with survival and overall response. The association between mRECIST response and survival was proven by a recent meta-analysis. ${ }^{35}$

\section{Balloon-occluded TACE (B-TACE)}

Recently, B-TACE has been developed in Japan. B-TACE is generally defined as the infusion of an emulsion of chemotherapeutic agents with lipiodol followed by gelatin particles under the occlu- 
sion of feeding arteries by a microballoon catheter which results in dense lipiodol emulsion accumulation in targeted nodules. ${ }^{36} \mathrm{Al}-$ though no high-grade evidence reports are comparing the therapeutic efficacy and the survival benefit between B-TACE and other TACE procedures, retrospective and small size studies have shown the superiority of therapeutic efficacy in B-TACE compared with that in CTACE. ${ }^{37,38}$ B-TACE is expected to be a promising TACE procedure for selected patients with BCLC stage B HCC.

\section{Drug-eluting beads TACE (DEB-TACE)}

TACE with calibrated doxorubicin-carrying microspheres (DCBeads) has been introduced as a novel device capable of ensuring more sustained and tumor-selective drug delivery and permanent embolization. ${ }^{39}$ Trials comparing DEB-TACE with cTACE have failed to show a survival benefit, but systemic toxicity from chemotherapy is reduced with DEB-TACE. The multi-center, randomized phase II PRECISION V trial indicated that DEB-TACE was better tolerated than CTACE, owing to a significant reduction of doxorubicin-related adverse events. ${ }^{40}$ TACE for HCC patients with Child-Pugh class $B$ is required to pay more attention to not only therapeutic efficacy but also liver-function impairment than for HCC patients with Child-Pugh class A. There is no RCT or meta-analysis comparing the efficacy and safety of DEB-TACE and CTACE in HCC patients with Child-Pugh class B. In several RCTs in favor of DEB-TACE, there was a better safety profile with a significant decrease in serious liver-related adverse events and systemic side effects compared with CTACE. ${ }^{41}$ In a retrospective cohort study, Shimose et al. recommended the choice of DEB-TCE for HCC patients with ChildPugh class $B$ due to the high incidence of arterio-portal shunt formation in patients with Child-Pugh class $A{ }^{42}$

\section{Systemic therapy}

In 2008, the SHARP trial assessing the multityrosine kinase inhibitor sorafenib (blocking VEGFR2, platelet-derived growth factor receptor [PDGFR], and Raf kinases) was the first to significantly improve survival. ${ }^{5}$ In 2018, based on the REFLECT trial, lenvatinib (blocking VEGFRs, fibroblast growth factor receptors, RET, KIT, and PDGFR A) demonstrated non-inferiority OS benefit versus sorafenib. ${ }^{43}$ Today, sorafenib and lenvatinib are available as a first-line MTAs for advanced HCC. Regorafenib (blocking VEGFRs, PDGFRs, KIT, and Tie2), cabozantinib (blocking VEGFRs, MET, and $A X L$ ), and ramucirumab (blocking VEGFR2) have been approved as a second-line treatment. All of these drugs are available for advanced HCC with Child-Pugh class A. Although these MTAs are usually selected for the treatment of BCLC stage $C$ HCC, the benefit of MTAs is even proven in a small subset of BCLC stage B HCC patients. ${ }^{44}$ Today, there are several therapeutic strategies for $B C L C$ stage B HCC in the real world, and we should select the most suitable treatment option to prolong the survival time and to keep the treatable condition as long as possible. The worst therapeutic scenario is to continue TACE for HCC with unsuitable TACE and to induce the deterioration of liver function.

\section{TACE FAILURE/REFRACTORINESS}

Multi-centric recurrence or intrahepatic metastasis of HCC during TACE requires additional TACE procedures. However, repeated TACE sometimes induces a decrease in therapeutic efficacy and deterioration of liver function. The criterion for TACE discontinuation is not yet fully defined. Bruix et al. ${ }^{45}$ proposed the concept of 'untreatable progression'. Untreatable progression includes major progression such as massive liver involvement, extrahepatic spread, and vascular invasion, but also minor intrahepatic progression with impaired liver function and performance status that contraindicate treatment. In particular, TACE should not be retreated as the following: 1) when it fails to achieve significant necrosis after two treatment sessions; 2) when follow-up treatment fails to induce significant tumor necrosis of progressed tumor sites; and 3) when the evaluation of the patient with progression prevents safe retreatment. ${ }^{45}$ On the other hand, for the objective determination of repeated TACE, the Assessment for Retreatment with TACE (ART) score and ABCR score was proposed. ${ }^{46,47}$ Although the clinical utility remains to be fully determined, these scores might support the decision of TACE discontinuation. In Japan, the concept of TACE failure/refractoriness was proposed. TACE failure/refractoriness was defined as follows: 1) an insufficient response after $\geq 2$ consecutive TACE procedures that is evident on response evaluation computed tomography or magnetic resonance imaging after 1-3 months, even after chemotherapeutic agents have been changed or the feeding artery has been reanalyzed; 2) the appearance of a higher number of lesions in the liver than that recorded in the previous TACE procedure (other than the nodule being treated); 3) the continuous elevation of tumor markers; 4) vascular invasion; and 5) extrahepatic spread. ${ }^{48}$ Although this concept is not well recognized worldwide, it is well accepted and utilized for the decision of TACE discontinuation in Japan. 


\section{TREATMENTS FOR TACE-UNSUITABILITY}

Kudo et al. ${ }^{49}$ classified BCLC stage B into following four groups in maximum tumor diameter showing poor response to CTACE; 1) 4-6 nodules and $\leq 3 \mathrm{~cm}$ in maximum tumor diameter showing good response to CTACE; 2) $<6$ nodules and $>3-6 \mathrm{~cm}$ in maxi- mum tumor diameter showing good response to CTACE; 3) out of up-to-7 criteria with multiple nodules $(\geq 7)$ showing poor response to $\mathrm{CTACE}$; 4$)<6$ nodules and $>6 \mathrm{~cm}$ in maximum tumor diameter showing poor response to CTACE (Fig. 2). Lenvatinib is the only first-line agent to demonstrate a survival benefit over TACE in TACE naïve patients out of up-to-7 criteria in a retrospective pro-

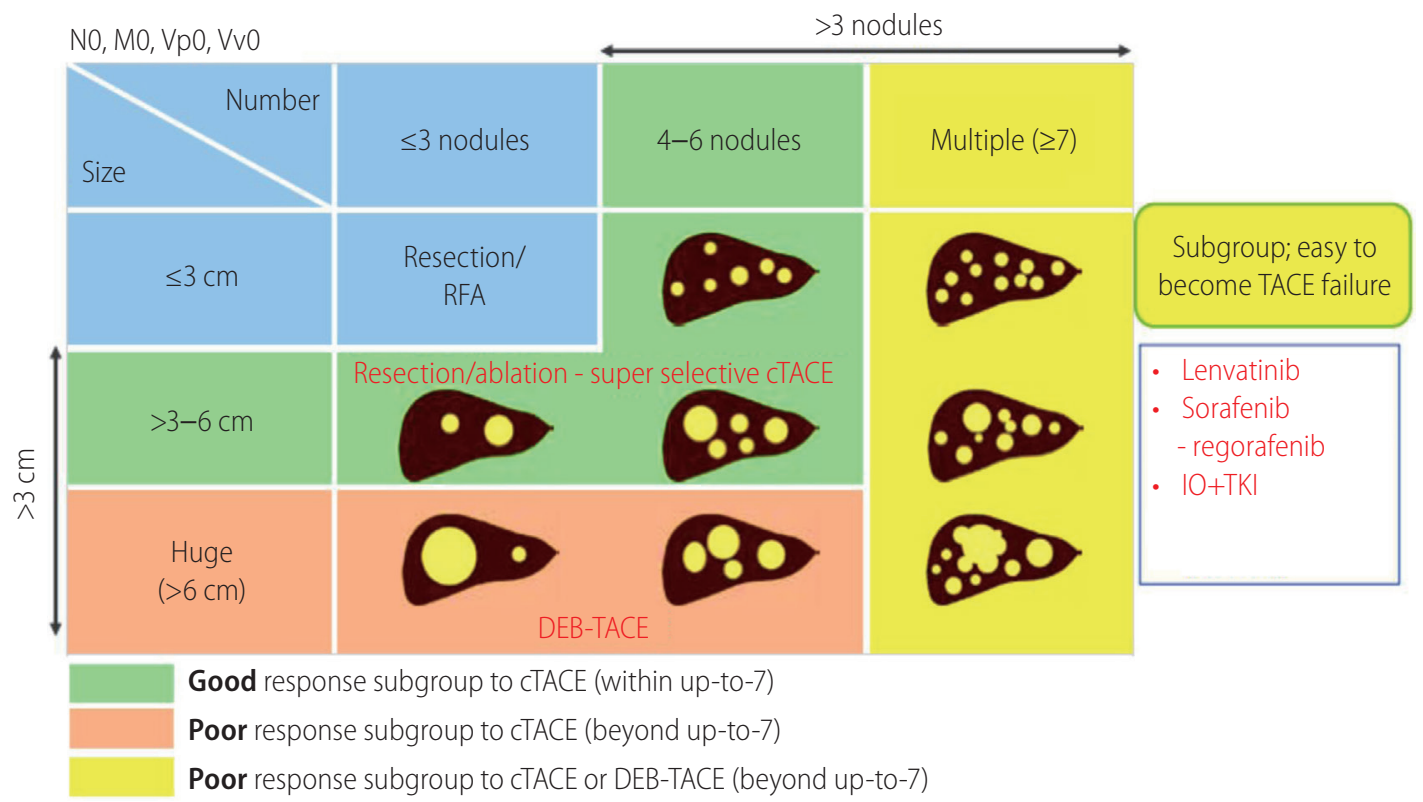

Figure 2. Grade of response to CTACE in BCLC stage B HCC. Reprinted from Bruix et al. ${ }^{45}$ with permission from S. Karger AG. RFA, radiofrequency ablation; CTACE, conventional transarterial chemoembolization; DEB-TACE, drug-eluting beads transarterial chemoembolization; IO, immune-oncology; TKI, tyrosine kinase inhibitor; BCLC, Barcelona Clinic Liver Cancer; HCC, hepatocellular carcinoma.

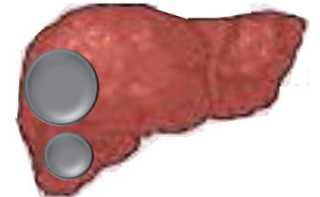

Hepatic resection Percutaneous treatment Radiation therapy

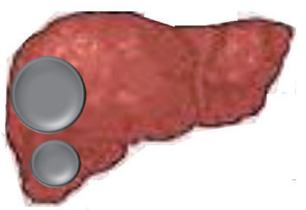

Percutaneous treatment TACE

Child-Pugh class A

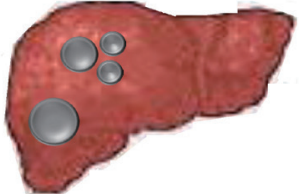

TACE, $\{$ MTA, $(I C I)\}$

TARE

Child-Pugh class B

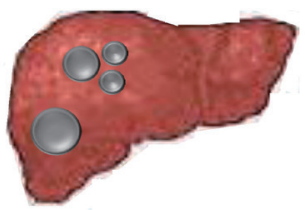

TACE

TARE

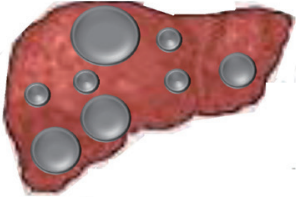

MTA, (ICI)

TARE

Figure 3. Potential therapeutic options in BCLC stage B. BCLC, Barcelona Clinic Liver Cancer; TACE, transarterial chemoembolization; MTA, molecular targeted agent; ICI, immune checkpoint inhibitor; TARE, transarterial radioembolization. 


\section{CLINICAL and MOLECULAR}

Volume_27 Number_2 April 2021

pensity score-matched study. ${ }^{50}$ Lenvatinib treatment seems to show favorable results in TACE-resistant HCC. In decision-tree analysis for OS in HCC patients with BCLC stage B, complete response (CR) by initial TACE was selected as the most important variable. In the decision-tree analysis for $C R,<3$ liver segments with nodules, simple nodular type, and within up-to-7 criteria, which are considered as suitable TACE criteria. ${ }^{51}$ Therefore, in patients who are ineligible for suitable TACE criteria, switching to MTAs before deterioration of liver function or TACE refractory might improve the prognosis than repeated TACE. ${ }^{52}$ A potential new therapeutic option in BCLC stage B is indicated in Figure 3.

\section{FUTURE PERSPECTIVES}

\section{The possibility of treatment with immune checkpoint inhibitors (ICls)}

The Food and Drug Administration (FDA) approved nivolumab (anti-programmed cell death protein-1 [PD-1] antibody) and pem- brolizumab (anti-PD-1 antibody) as an adjunct treatment for HCC patients after sorafenib failure, in 2017 and 2018, respectively. The FDA approved ipilimumab (CTLA-4 antibody) and nivolumab as a combination therapy in 2019. Many clinical trials with ICls are ongoing. Unfortunately, phase 3 trials comparing nivolumab with sorafenib in front-line and pembrolizumab with placebo in second-line resulted in negative results. In 2019, the IMbrave-150 trial with atezolizumab (anti-PD-L1 antibody) and bevacizumab (anti-VEGF antibody) showed superiority in prolonged OS and progression free survival compared with sorafenib. ${ }^{6}$ The reasons why the combination therapy with atezolizumab and bevacizumab showed positive results are not fully clarified.

HCC contains a high level of VEGF. In addition to its potent angiogenic effect, VEGF enhances the migration of cytotoxic T cells to tumor tissue, induces the secretion of interleukin-10 by myeloid-derived suppressor cells, and suppresses the maturation of dendritic cells (DCs) as well as the cytotoxic activity of T cells. In addition, VEGF enhances the migration and proliferation of Tregs through VEGF receptor-2. Tregs suppress the maturation of DCS and the cytotoxic activity of T cells. VEGF directly inhibits the pro-

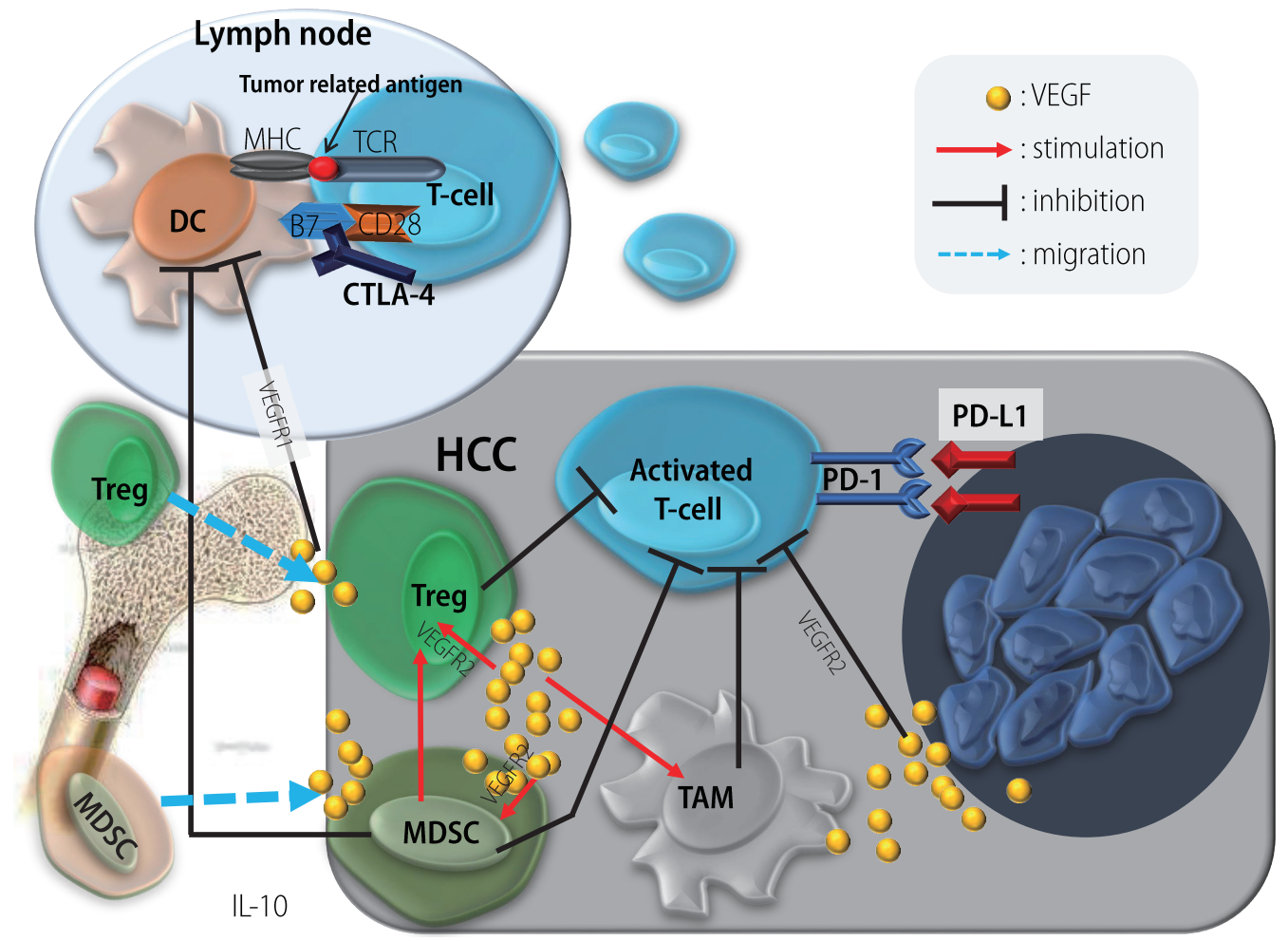

Figure 4. Influence of VEGF on tumor immunity. MHC, major histocompatibility complex; TCR, T-cell receptor; DC, dendritic cell; CTLA-4, cytotoxic Tlymphocyte antigen 4; VEGF, vascular endothelial growth factor; Treg, regulatory T cell; MDSC, myeloid-derived suppressor cell; IL, interleukin; HCC, hepatocellular carcinoma; TAM, tumor associated macrophage; PD-1, programmed death-1; PD-L1, programmed death ligand 1. 
liferation and cytotoxic activity of T cells through VEGF receptor-2 on T cells and inhibits the maturation of DCs through VEGF receptor-1 in DCs (Fig. 4). ${ }^{52}$ These findings clearly indicate that VEGF has an immunosuppressive function. In combination therapy with atezolizumab and bevacizumab, bevacizumab might support the function of atezolizumab by suppressing the immunosuppressive function of VEGF. To clarify the precise mechanism of tumor immunity will develop a more potent ICIs in HCC.

\section{Evaluation of liver function}

Child-Pugh classification is used to evaluate liver function worldwide. In the BCLC staging system, liver function was assessed using the Child-Pugh classification. Recently, the albuminbilirubin (ALBI) grading system has been shown to stratify HCC patients across BCLC stages. ${ }^{53}$ Several studies have reported the superiority of $A L B I$ grade over Child-Pugh classification to assess liver function for therapeutic decision making. ${ }^{54}$ Further investigation will be required to clarify a better assessment system of liver function for the selection of optimal treatment for HCC with BCLC stage B.

\section{Subclassification of BCLC stage B according to genetic alterations}

Mutations in the TERT promoter region are the most frequent genetic alterations (60\%) followed by TP53 (30\%), CTNNB1 $(30 \%)$, and AXIN $1(10 \%)$. HCC is among the solid cancers with the fewest somatic mutations that can be targeted with MTAs. ${ }^{55}$ However, an integrative genomic analysis may enable the stratification between an active immune class and resistance to $\mathrm{ICl}$ class characterized by activation of the $\mathrm{Wnt} /$ - $\beta$-catenin pathway. ${ }^{56,57}$

\section{CONCLUSIONS}

BCLC stage B consists of patients with heterogeneous HCC (from slightly above the Milan criteria to large/multifocal tumor burden). The standard recommended treatment of BCLC stage B is TACE. However, hepatic resection, liver transplantation, radiation therapy, and percutaneous therapy have become to be selected for the treatment option of left-hand side on the BCLC stage $B$ to prolong survival time in a real world. TACE has also progressed and prolonged the survival time. However, repeated TACE reduces the treatment effect and induces liver function im- pairment. Under these circumstances, recent progress of MTAs and ICls will cause a drastic paradigm change for the treatment of right-hand side on BCLC stage $\mathrm{B}$.

\section{Authors' contribution}

Takuji Torimura and Hideki Iwamoto were involved in the writing of the article.

\section{Conflicts of Interest}

The authors have no conflicts to disclose.

\section{REFERENCES}

1. Bray F, Ferlay J, Soerjomataram I, Siegel RL, Torre LA, Jemal A. Global cancer statistics 2018: GLOBOCAN estimates of incidence and mortality worldwide for 36 cancers in 185 countries. CA Cancer J Clin 2018;68:394-424.

2. Villanueva A. Hepatocellular carcinoma. N Engl J Med 2019;380: 1450-1462.

3. Lozano R, Naghavi M, Foreman K, Lim S, Shibuya K, Aboyans V, et al. Global and regional mortality from 235 causes of death for 20 age groups in 1990 and 2010: a systematic analysis for the Global Burden of Disease Study 2010. Lancet 2012;380:2095-2128.

4. Yin L, Li H, Li AJ, Lau WY, Pan ZY, Lai EC, et al. Partial hepatectomy vs. transcatheter arterial chemoembolization for resectable multiple hepatocellular carcinoma beyond Milan criteria: a RCT. J Hepatol 2014:61:82-88

5. Llovet JM, Ricci S, Mazzaferro V, Hilgard P, Gane E, Blanc JF, et al. Sorafenib in advanced hepatocellular carcinoma. N Engl J Med 2008;359:378-390.

6. Finn RS, Qin S, Ikeda M, Galle PR, Ducreux M, Kim TY, et al. Atezolizumab plus bevacizumab in unresectable hepatocellular carcinoma. N Engl J Med 2020;382:1894-1905.

7. Llovet JM, Brú C, Bruix J. Prognosis of hepatocellular carcinoma: the BCLC staging classification. Semin Liver Dis 1999;19:329-338.

8. Forner A, Reig M, Bruix J. Hepatocellular carcinoma. Lancet 2018; 391:1301-1314

9. Bolondi L, Burroughs A, Dufour JF, Galle PR, Mazzaferro V, Piscaglia $F$, et al. Heterogeneity of patients with intermediate (BCLC B) hepatocellular carcinoma: proposal for a subclassification to facilitate treatment decisions. Semin Liver Dis 2012;32:348-359.

10. Kudo M, Arizumi T, Ueshima K, Sakurai T, Kitano M, Nishida N. Subclassification of BCLC B stage hepatocellular carcinoma and treatment strategies: proposal of modified Bolondi's subclassification (Kinki Criteria). Dig Dis 2015;33:751-758.

11. Ciria R, López-Cillero P, Gallardo AB, Cabrera J, Pleguezuelo M, Ayl- 
lón $M D$, et al. Optimizing the management of patients with $B C L C$ stage-B hepatocellular carcinoma: modern surgical resection as a feasible alternative to transarterial chemoemolization. Eur J Surg Oncol 2015;41:1153-1161.

12. Hyun MH, Lee YS, Kim JH, Lee CU, Jung YK, Seo YS, et al. Hepatic resection compared to chemoembolization in intermediate- to advanced-stage hepatocellular carcinoma: a meta-analysis of highquality studies. Hepatology 2018;68:977-993.

13. Degroote H, Gallebout E, lesari S, Dekervel J, Schreiber J, Pirenne J, et al. Extended criteria for liver transplantation in hepatocellular carcinoma. A retrospective, multicentric validation study in Belgium. Surg Oncol 2020;33:231-238.

14. Lingiah VA, Niazi M, Olivo R, Paterno F, Guarrera JV, Pyrsopoulos NT. Liver transplantation beyond Milan criteria. J Clin Transl Hepatol 2020;8:69-75.

15. Yao FY, Xiao L, Bass NM, Kerlan R, Ascher NL, Roberts JP. Liver transplantation for hepatocellular carcinoma: validation of the UCSF-expanded criteria based on preoperative imaging. Am J Transplant 2007;7:2587-2596.

16. Ho EY, Cozen ML, Shen H, Lerrigo R, Trimble E, Ryan JC, et al. Expanded use of aggressive therapies improves survival in early and intermediate hepatocellular carcinoma. HPB (Oxford) 2014;16:758767.

17. Farinati F, Vanin V, Giacomin A, Pozzan C, Cillo U, Vitale A, et al. $B C L C$ stage $B$ hepatocellular carcinoma and transcatheter arterial chemoembolization: a 20-year survey by the Italian Liver Cancer group. Liver Int 2015;35:223-231.

18. Dobrzycka M, Spychalski P, Rostkowska O, Wilczyński M, Kobiela $P, G r a ̨ t ~ M$, et al. Stereotactic body radiation therapy for early-stage hepatocellular carcinoma - a systematic review on outcome. Acta Oncol 2019;58:1706-1713.

19. Mazzaferro V, Regalia E, Doci R, Andreola S, Pulvirenti A, Bozzetti $F$, et al. Liver transplantation for the treatment of small hepatocellular carcinomas in patients with cirrhosis. N Engl J Med 1996;334:693699.

20. Yang JF, Lo CH, Huang WY. Is stereotactic body radiotherapy better than radiofrequency ablation for the treatment of hepatocellular carcinoma? J Clin Oncol 2016;34:2797.

21. Kim N, Cheng J, Jung I, Liang J, Shih YL, Huang WY, et al. Stereotactic body radiation therapy vs. radiofrequency ablation in Asian patients with hepatocellular carcinoma. J Hepatol 2020;73:121-129.

22. Sangro B, Iñarrairaegui M, Bilbao Jl. Radioembolization for hepatocellular carcinoma. J Hepatol 2012;56:464-473.

23. Salam R, Lewandowski RJ, Mulcahy MF, Riaz A, Ryu RK, Ibrahim $\mathrm{S}$, et al. Radioembolization for hepatocellular carcinoma using Yttrium-90 microspheres: a comprehensive report of long-term outcomes. Gastroenterology 2010;138:52-64.

24. Vilgrain V, Pereira $H$, Assenat E, Guiu B, Ilonca AD, Pageaux GP, et al. Efficacy and safety of selective internal radiotherapy with yttrium-90 resin microspheres compared with sorafenib in locally advanced and inoperable hepatocellular carcinoma (SARAH): an open-label randomised controlled phase 3 trial. Lancet Oncol 2017;18:1624-1636.

25. Chow PKH, Gandhi M, Tan SB, Khin MW, Khasbazar A, Ong J, et al. SIRveNIB: selective internal radiation therapy versus sorafenib in Asia-Pacific patients with hepatocellular carcinoma. J Clin Oncol 2018;36:1913-1921.

26. Chen LT, Martinelli E, Cheng AL, Pentheroudakis G, Qin S, Bhattacharyya GS, et al. Pan-Asian adapted ESMO clinical practice guidelines for the management of patients with intermediate and advanced/relapsed hepatocellular carcinoma: a TOS-ESMO initiative endorsed by CSCO, ISMPO, JSMO, KSMO, MOS and SSO. Ann Oncol 2020;31:334-351.

27. Yamada R, Sato M, Kawabata M, Nakatsuka H, Nakamura K, Takashima S. Hepatic artery embolization in 120 patients with unresectable hepatoma. Radiology 1983;148:397-401.

28. Verslype C, Rosmorduc O, Rougier P; ESMO Guidelines Working Group. Hepatocellular carcinoma: ESMO-ESDO clinical practice guidelines for diagnosis, treatment and follow-up. Ann Oncol 2012;23 Suppl 7:vi41-vii48.

29. Llovet JM, Real MI, Montaña X, Planas R, Coll S, Aponte J, et al. Arterial embolisation or chemoembolisation versus symptomatic treatment in patients with unresectable hepatocellular carcinoma: a randomised controlled trial. Lancet 2002;359:1734-1739.

30. Llovet JM, Bruix J. Systematic review of randomized trials for unresectable hepatocellular carcinoma: chemoembolization improves survival. Hepatology 2003;37:429-442.

31. Takayasu K, Arii S, Kudo M, Ichida T, Matsui O, Izumi N, et al. Superselective transarterial chemoembolization for hepatocellular carcinoma. Validation of treatment algorithm proposed by Japanese guidelines. J Hepatol 2012;56:886-892.

32. Forner A, Gilabert M, Bruix J, Raoul JL. Treatment of intermediatestage hepatocellular carcinoma. Nat Rev Clin Oncol 2014;11:525535.

33. European Association For The Study Of The Liver; European Organisation For Research And Treatment Of Cancer. EASL-EORTC clinical practice guidelines: management of hepatocellular carcinoma. J Hepatol 2012;56:908-943.

34. Raoul JL, Sangro B, Forner A, Mazzaferro V, Piscaglia F, Bolondi L, et al. Evolving strategies for the management of intermediate-stage hepatocellular carcinoma: available evidence and expert opinion on the use of transarterial chemoembolization. Cancer Treat Rev 2011;37:212-220.

35. Vincenzi B, Di Maio M, Silletta M, D'Onofrio L, Spoto C, Piccirillo $M C$, et al. Prognostic relevance of objective response according to EASL criteria and mRECIST criteria in hepatocellular carcinoma pa- 
tients treated with loco-regional therapies: a literature-based metaanalysis. PLoS One 2015;10:e0133488.

36. Irie T, Kuramochi M, Takahashi N. Dense accumulation of lipiodol emulsion in hepatocellular carcinoma nodule during selective balloon-occluded transarterial chemoembolization: measurement of balloon-occluded arterial stump pressure. Cardiovasc Intervent Radiol 2013;36:706-713.

37. Irie T, Kuramochi M, Kamoshida T, Takahashi N. Selective balloonoccluded transarterial chemoembolization for patients with one or two hepatocellular carcinoma nodules: retrospective comparison with conventional super-selective TACE. Hepatol Res 2016;46:209214.

38. Shirono $T$, Iwamoto $H$, Niizeki $T$, Shimose $S$, Nakano $M$, Satani $M$, et al. Epirubicin is more effective than miriplatin in balloon-occluded transcatheter arterial chemoembolization for hepatocellular carcinoma. Oncology 2019;96:79-86.

39. Golfieri R, Giampalma E, Renzulli M, Cioni R, Bargellini I, Bartolozzi $C$, et al. Randomised controlled trial of doxorubicin-eluting beads vs conventional chemoembolisation for hepatocellular carcinoma. Br J Cancer 2014;111:255-264.

40. Lammer J, Malagari K, Vogl T, Pilleul F, Denys A, Watkinson A, et al. Prospective randomized study of doxorubicin-eluting-bead embolization in the treatment of hepatocellular carcinoma: results of the PRECISION V study. Cardiovasc Intervent Radiol 2010;33:41-52.

41. Facciorusso A. Drug-eluting beads transarterial chemoembolization for hepatocellular carcinoma: current state of the art. World J Gastroenterol 2018;24:161-169.

42. Shimose $S$, Iwamoto $H$, Tanaka M, Niizeki T, Shirono T, Nakano M, et al. Increased arterio-portal shunt formation after drug-eluting beads TACE for hepatocellular carcinoma. Oncology 2020;98:558-565.

43. Kudo M, Finn RS, Qin S, Han KH, Ikeda K, Piscaglia F, et al. Lenvatinib versus sorafenib in first-line treatment of patients with unresectable hepatocellular carcinoma: a randomised phase 3 noninferiority trial. Lancet 2018;391:1163-1173.

44. Bruix J, Raoul JL, Sherman M, Mazzaferro V, Bolondi L, Craxi A, et al. Efficacy and safety of sorafenib in patients with advanced hepatocellular carcinoma: subanalyses of a phase III trial. J Hepatol 2012; $57: 821-829$

45. Bruix j, Sherman M; American Association for the Study of Liver Diseases. Management of hepatocellular carcinoma: an update. Hepatology 2011;53:1020-1022.

46. Sieghart W, Hucke F, Pinter M, Graziadei I, Vogel W, Müller C, et al. The ART of decision making: retreatment with transarterial chemoembolization in patients with hepatocellular carcinoma. Hepatology 2013:57:2261-2273.
47. Hucke F, Sieghart W, Pinter M, Graziadei I, Vogel W, Müller C, et al. The ART-strategy: sequential assessment of the ART score predicts outcome of patients with hepatocellular carcinoma re-treated with TACE. J Hepatol 2014;60:118-126.

48. Kudo M, Matsui O, Izumi N, Kadoya M, Okusaka T, Miyayama S, et al. Transarterial chemoembolization failure/refractoriness: JSHLCSGJ criteria 2014 update. Oncology 2014;87 Suppl 1:22-31.

49. Kudo M, Han KH, Ye SL, Zhou J, Huang YH, Lin SM, et al. A changing paradigm for the treatment of intermediate-stage hepatocellular carcinoma: Asia-Pacific primary liver cancer expert consensus statements. Liver Cancer 2020;9:245-260.

50. Shimose S, Kawaguchi T, Tanaka M, Iwamoto H, Miyazaki K, Mori-

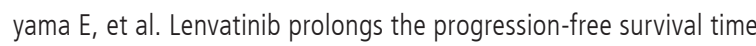
of patients with intermediate-stage hepatocellular carcinoma refractory to transarterial chemoembolization: a multicenter cohort study using data mining analysis. Oncol Lett 2020;20:2257-2265.

51. Shimose S, Kawaguchi T, Iwamoto H, Niizeki T, Shirono T, Tanaka $M$, et al. Indication of suitable transarterial chemoembolization and multikinase inhibitors for intermediate stage hepatocellular carcinoma. Oncol Lett 2020;19:2667-2676.

52. Chouaib S, Messai Y, Couve S, Escudier B, Hasmim M, Noman MZ. Hypoxia promotes tumor growth in linking angiogenesis to immune escape. Front Immunol 2012;3:21.

53. Johnson PJ, Berhane S, Kagebayashi C, Satomura S, Teng M, Reeves $\mathrm{HL}$, et al. Assessment of liver function in patients with hepatocellular carcinoma: a new evidence-based approach-the ALBI grade. J Clin Oncol 2015;33:550-558.

54. Hiraoka A, Kumada T, Kudo M, Hirooka M, Tsuji K, Itobayashi E, et al. Albumin-bilirubin (ALBI) grade as part of the evidence-based clinical practice guideline for HCC of the Japan society of hepatology: a comparison with the liver damage and Child-Pugh classifications. Liver Cancer 2017;6:204-215.

55. Zehir A, Benayed R, Shah RH, Syed A, Middha S, Kim HR, et al. Mutational landscape of metastatic cancer revealed from prospective clinical sequencing of 10,000 patients. Nat Med 2017;23:703-713.

56. Sia D, Jiao Y, Martinez-Quetglas I, Kuchuk O, Villacorta-Martin C, Castro de Moura $\mathrm{M}$, et al. Identification of an immune-specific class of hepatocellular carcinoma, based on molecular features. Gastroenterology 2017;153:812-826.

57. Harding JJ, Nandakumar S, Armenia J, Khalil DN, Albano M, Ly M, et al. Prospective genotyping of hepatocellular carcinoma: clinical implications of next-generation sequencing for matching patients to targeted and immune therapies. Clin Cancer Res 2019;25:21162126. 